\title{
Stability of hydrogen-antihydrogen mixtures at low energies
}

\section{Citation}

Jonsell, Svante, Alejandro Saenz, Piotr Froelich, Bernard Zygelman, and Alexander Dalgarno. 2001. "Stability of Hydrogen-Antihydrogen Mixtures at Low Energies." Physical Review A 64 (5) (October 12). doi:10.1103/physreva.64.052712.

\section{Published Version}

doi:doi.org/10.1103/PhysRevA.64.052712

\section{Permanent link}

http://nrs.harvard.edu/urn-3:HUL.InstRepos:30407683

\section{Terms of Use}

This article was downloaded from Harvard University's DASH repository, and is made available under the terms and conditions applicable to Other Posted Material, as set forth at http:// nrs.harvard.edu/urn-3:HUL.InstRepos:dash.current.terms-of-use\#LAA

\section{Share Your Story}

The Harvard community has made this article openly available.

Please share how this access benefits you. Submit a story.

\section{Accessibility}




\title{
Stability of hydrogen-antihydrogen mixtures at low energies
}

\author{
Svante Jonsell, ${ }^{1,2}$ Alejandro Saenz, ${ }^{3, *}$ Piotr Froelich, ${ }^{1}$ Bernard Zygelman, ${ }^{4}$ and Alexander Dalgarno ${ }^{5}$ \\ ${ }^{1}$ Department of Quantum Chemistry, Uppsala University, Box 518, SE-75120 Uppsala, Sweden \\ ${ }^{2}$ NORDITA, Blegdamsvej 17, DK-2100 Copenhagen, Denmark \\ ${ }^{3}$ Max-Planck-Institute for Quantum Optics, Hans-Kopfermann-Str. 1, D-85748 Garching, Germany \\ ${ }^{4}$ Department of Physics, University of Nevada, Las Vegas, Nevada 89154 \\ ${ }^{5}$ Harvard - Smithsonian Center for Astrophysics, 60 Garden Street, Cambridge, Massachusetts 02138
}

(Received 20 May 2000; revised manuscript received 15 June 2001; published 12 October 2001)

\begin{abstract}
The stability of antimatter in contact with matter has been investigated. The interaction between hydrogen and antihydrogen is considered as the prototype reaction. We have focused interest on the rates for protonantiproton and/or electron-positron annihilation during hydrogen-antihydrogen collisions at low energies. In particular, we have concentrated on the calculation of the rates for the rearrangement reaction leading to formation of protonium and positronium, ending inevitably in particle-antiparticle annihilation. The cross section for the rearrangement collision has been calculated in a fully quantum-mechanical treatment. Additionally, we have calculated cross sections for direct annihilation during the collision process, which was found to be comparable to the rearrangement cross section. The elastic cross section and its low-energy limit, given by the scattering length, have been calculated, allowing, by comparison to the inelastic processes, a prediction for the efficiency of cooling antihydrogen via collisions with ultracold hydrogen atoms.
\end{abstract}

DOI: $10.1103 /$ PhysRevA.64.052712

PACS number(s): $36.10 .-\mathrm{k}, 34.90 .+\mathrm{q}$

\section{INTRODUCTION}

In the present paper, we investigate the stability of antimatter in contact with ordinary matter. The collisional interaction between hydrogen $\mathrm{H}$ and antihydrogen $\overline{\mathrm{H}}$ is considered as the prototype reaction. The problem has received limited attention in the past [1-7] but is rapidly gaining interest as recent advances in producing, trapping, and cooling antiprotons and positrons opened the possibility of antihydrogen formation at low temperatures. This may allow studies of antimatter and tests of fundamental physical principles such as the $C P T$ invariance or the weak equivalence principle for antiparticles. Such experiments are in progress at CERN AD (Antiproton Decelerator), within the Apparatus for High Precision Experiments on Neutral Antimatter (ATHENA), Antihydrogen Trap (ATRAP), and Atomic Spectroscopy and Collisions Using Slow Antiprotons (ASACUSA) collaborations.

The ATHENA $[8,9]$ and ATRAP [10] experiments aim at the production of antihydrogen atoms at low energies, capturing these atoms in a magnetic trap, and comparing the energy levels of antihydrogen with those of hydrogen. ASACUSA will carry out a program on antiprotoncontaining atoms and atomic physics with antiprotons, notably on antiprotons in collisions and high-precision spectroscopy on antiprotonic species [11].

The prospects of laboratory experiments with cold antihydrogen reinforce the importance of questions regarding the stability of antimatter in contact with ordinary matter. Additionally, these questions are of interest in astrophysics, particularly in the context of the matter-antimatter asymmetry in the universe, and in interpretations of distortions of the cos-

\footnotetext{
*Present address: Fachbereich Chemie, Universität Konstanz, Fach M 721, D-78457 Konstanz, Germany.
}

mic background radiation due to relic $\gamma$ rays from matterantimatter annihilation.

In this paper, we present a detailed investigation of the relevant cross sections and scattering lengths for a pair of interacting hydrogen-antihydrogen atoms in their ground states. In particular, we investigate the possibility of cooling antihydrogen via contact with cold hydrogen, a method first suggested by Shlyapnikov et al. [5]. In the collision of an antihydrogen atom with a hydrogen atom, where both atoms are in their ground state, elastic scattering leads to thermalization and is responsible for the cooling. The feasibility of collisional cooling depends on the rate of thermalization of antihydrogen compared to its annihilation rate. In a recent paper [12], we have presented results for particle-antiparticle annihilation, through the rearrangement process, which results in the production of positronium $\mathrm{Ps} \equiv e^{+} e^{-}$and protonium $\mathrm{Pn} \equiv p \bar{p}$ according to $\mathrm{H}+\overline{\mathrm{H}} \rightarrow p \bar{p}+e^{+} e^{-}$. The annihilation then occurs within the bound particle-antiparticle pairs that are formed. Another loss process is annihilation "in flight," directly from the scattering states of the colliding system during the collision. Hence, both the strong nuclear force and the Coulomb force give rise to inelastic processes. Both inelastic channels result in losses of antihydrogen, and thus, compete with the desired cooling process. Therefore, a knowledge of the rates for the elastic and inelastic collisions, is of paramount importance.

At low energies $E$, the elastic cross section $\sigma^{\text {el }}$ is constant, while the inelastic cross sections vary as $1 / \sqrt{E}$ [13]. For a thermalized sample described by a Maxwell-Boltzmann distribution, this implies that the rate $\lambda_{\mathrm{el}}$ for elastic collisions behaves as $\lambda_{\mathrm{el}} \sim \sqrt{T}$ (where $T$ is the temperature), while the rate $\lambda_{\text {inel }}$ for inelastic collisions is constant. Hence, there must exist a certain limiting temperature below which $\lambda_{\text {inel }}$ $>\lambda_{\mathrm{el}}$, and consequently the loss of antihydrogen dominates over the cooling process. The ratio $\lambda_{\text {inel }} / \lambda_{\text {el }}$ determines the 
lowest temperature attainable in the cooling of antihydrogen.

In the present paper, we mainly concentrate on the calculation of the scattering cross section for the inelastic rearrangement reaction leading to annihilation via the formation of protonium and positronium in bound states. This process will be discussed in the following section. In Sec. III, annihilation "in flight" will be discussed. This will be followed by a calculation of the elastic cross section in Sec. IV. A discussion of the results with respect to their importance for the design and possible implementation of an antihydrogen cooling experiment follows in Sec. V. We will use atomic units except where we explicitly state otherwise. In order to give a sense of the corresponding scale of temperature, we will sometimes express the kinetic energy of the antihydrogen atoms in degrees Kelvin, defined by dividing the energy by Boltzmann's constant. When expressed in degrees Kelvin, the kinetic energy will always refer to the frame where the average velocity of the atoms is zero, i.e., the laboratory frame.

\section{THE REARRANGEMENT PROCESS}

In this section, the rearrangement reaction

$$
\mathrm{H}(1 s)+\overline{\mathrm{H}}(1 s) \rightarrow \operatorname{Pn}(N L M)+\operatorname{Ps}(n l m)
$$

will be considered, i.e., the collision of a hydrogen and an antihydrogen atom (both being in their $1 s$ ground states) yielding protonium and positronium left in the states NLM and $n l m$, respectively. In this case $N$ and $n$ denote the principal quantum numbers, $L$ and $l$ the angular momentum quantum numbers, and $M$ and $m$ the magnetic quantum numbers. Since the two leptons are not identical particles, the Pauli principle imposes no restriction on the allowed states of the system. One may thus choose to express the spin of the leptons either in terms of their total spin, or in terms of the individual spins of the leptons. The spatial wave function is independent of the spin state, so all the different spin states will be degenerate. Therefore, we will omit the spin quantum numbers below. The same is true for the hadronic (i.e., proton and antiproton) spins.

\section{A. Energy considerations}

We will work in the center-of-mass frame, where the asymptotic relative momenta in the initial and final channel are $\mathbf{k}_{i}$ and $\mathbf{k}_{f}$, respectively. The energies $E_{i}$ and $E_{f}$ in the initial and final channel can most easily be obtained from their asymptotic values. In this way one obtains

$$
\begin{gathered}
E_{i}=\epsilon_{i}+\mathcal{E}_{1 s}^{\mathrm{H}}+\mathcal{E}_{1 s}^{\overline{\mathrm{H}}}=\epsilon_{i}-\frac{1}{2}-\frac{1}{2}=\frac{k_{i}^{2}}{2 \mu_{i}}-1, \\
E_{f}=\epsilon_{f}+\mathcal{E}_{N}^{\mathrm{Pn}}+\mathcal{E}_{n}^{\mathrm{Ps}}=\frac{k_{f}^{2}}{2 \mu_{f}}-\frac{m_{p}}{4 N^{2}}-\frac{1}{4 n^{2}},
\end{gathered}
$$

where $\mathcal{E}$ denotes internal energies, $\epsilon$ external, translational energies, and $\mu$ the reduced mass of the channel fragments.

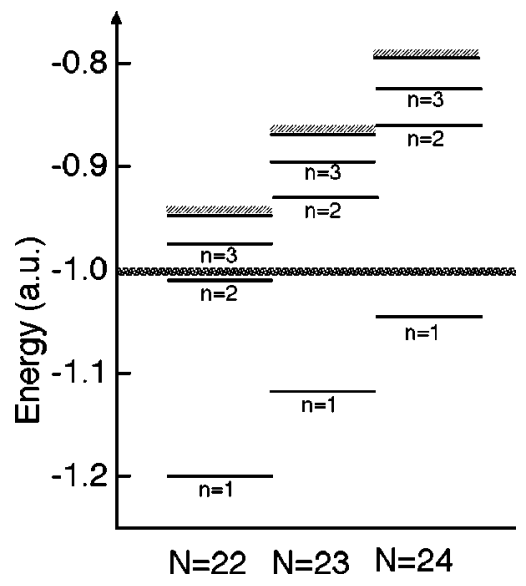

FIG. 1. Scattering thresholds with Pn principal quantum number $N=22-24$, and positronium principal quantum number $n$. The lines show the sum of the protonium and positronium binding energies. Any final state with energy below -1 a.u., corresponding to zero collision energy, can be formed in zero-energy hydrogenantihydrogen collisions.

In the present case, $\mu_{i}=m_{\mathrm{H}} / 2 \simeq m_{p} / 2$ and $\mu_{f}$ $=\left(m_{\mathrm{Pn}} m_{\mathrm{Ps}}\right) /\left(m_{\mathrm{Pn}}+m_{\mathrm{Ps}}\right) \simeq m_{\mathrm{Ps}} \simeq 2$.

Conservation of the total energy yields

$$
\epsilon_{i}-1=\epsilon_{f}+\mathcal{E}_{N}^{\mathrm{Pn}}+\mathcal{E}_{n}^{\mathrm{Ps}} .
$$

For cold collisions, the initial energy $\epsilon_{i}$ is very much smaller than all other relevant energies, and can thus be neglected in the energy balance. Since for the final channel to be open it is required that $\epsilon_{f} \geqslant 0$, Eq. (4) reduces to

$$
\mathcal{E}_{n}^{\mathrm{Ps}}+\mathcal{E}_{N}^{\mathrm{Pn}}=-\frac{1}{4 n^{2}}-\frac{m_{p}}{4 N^{2}} \leqslant-1 \Rightarrow N \leqslant \sqrt{\frac{m_{p}}{4-1 / n^{2}}}
$$

The relation (5) restricts the possible values of the principal quantum numbers $N$ and $n$. The largest allowed value of $N$ is obtained for $n=1$, i.e., $\mathcal{E}_{1 s}^{\mathrm{Ps}}=-0.25$. In this case, the maximum principal quantum number in which protonium can be formed is $N_{\max }=24$, while the rearrangement channel is closed if $N>N_{\max }=24$. In the interval $24 \geqslant N \geqslant 22$, only bound states of positronium are energetically accessible. In fact, only two principal quantum numbers, namely, $n=1$ and $n=2$, can occur for the positronium atom in this case. For $N<22$, either a bound state (with arbitrary $n$ ) or a free $e^{+}-e^{-}$pair can be formed. The structure of the final-state binding energies is displayed in Fig. 1.

Returning to Eq. (4), with $\epsilon_{i} \approx 0$, we find that the energy released in the reaction (1) is converted to kinetic energy of translational motion in the final channel $\epsilon_{f}$. Due to the large mass difference between protonium and positronium $\left(\mu_{f}\right.$ $\simeq m_{\mathrm{Ps}}$ ), this energy will, to a very good approximation, be left in the translational motion of the positronium alone. The minimum amount of energy that has to be transferred to the kinetic energy of the positronium atom is listed together with the corresponding value of $N_{\max }$ in Table I for different values of $n$. 
TABLE I. Maximum protonium excitation $N_{\max }$ and minimum amount of kinetic energy $\epsilon_{f \text {, min }}$ that has to be transfered to the positronium for different final positronium excitations $n$.

\begin{tabular}{lcc}
\hline \hline$n$ & $N_{\max }$ & $\boldsymbol{\epsilon}_{f, \min }$ \\
\hline 1 & 24 & 0.04695 \\
2 & 22 & 0.01094 \\
3 & 21 & 0.06869 \\
4 & 21 & 0.05654 \\
$\infty$ & 21 & 0.04091 \\
\hline \hline
\end{tabular}

\section{B. Formulation of the scattering process}

\section{The cross section}

The cross section for the rearrangement process in Eq. (1) is

$$
\sigma^{\mathrm{rearr}}=\frac{(2 \pi)^{4}}{k_{i}^{2}} \sum_{f} \delta\left(E_{f}-E_{i}\right)\left|T_{f i}\right|^{2}
$$

where $f$ stands for the complete set of quantum numbers specifying the final state of protonium and positronium, each in a specific internal state, and moving with a specific kinetic energy, in a specific direction. The main task in the determination of the rearrangement cross section is the evaluation of the transition matrix element $T_{f i}$, which is given by

$$
T_{f i}=\left\langle\Upsilon_{f}\left|\hat{V}_{f}\right| \Psi_{\mathbf{k}_{i}}^{(+)}\right\rangle
$$

The wave function $\Psi_{\mathbf{k}_{i}}^{(+)}$is the solution of the complete Hamiltonian describing an interacting hydrogenantihydrogen system with total energy $E_{i}$, that fulfills the boundary condition for incoming hydrogen and antihydrogen atoms. The wave function $Y_{f}$ is the solution of the eigenvalue problem

$$
\hat{\mathrm{H}}_{f} \Upsilon_{f}=E_{f} \Upsilon_{f}
$$

where $\hat{H}_{f}$ is the final-channel Hamiltonian describing a noninteracting protonium-positronium system. This corresponds to a final-channel partitioning of the complete Hamiltonian $\hat{H}$ according to $\hat{\mathrm{H}}=\hat{\mathrm{H}}_{f}+\hat{\mathrm{V}}_{f}$, where

$$
\hat{\mathrm{V}}_{f}=-\frac{1}{r_{p e}}+\frac{1}{r_{p \bar{e}}}+\frac{1}{r_{p e}^{-}}-\frac{1}{r_{p e}^{-}}
$$

describes the interaction between protonium and positronium. Here, and in the following, the notation $r_{i j}=\left|\mathbf{r}_{j}-\mathbf{r}_{i}\right|$ is adopted for denoting the distance between particles $i$ and $j$.

\section{The initial- and final-state wave functions}

The total scattering wave function $\Psi_{\mathbf{k}_{i}}^{(+)}$may be simplified by using the distorted-wave approximation in which $\Psi_{\mathbf{k}_{i}}^{(+)}$is replaced by an incoming distorted wave represented by the Born-Oppenheimer approximation

$$
\Psi_{\mathbf{k}_{i}}^{(+)}\left(\mathbf{R} ; \mathbf{r}_{e}, \mathbf{r}_{e}^{-}\right)=\psi_{i}\left(R ; \mathbf{r}_{e}, \mathbf{r}_{e}^{-}\right) \chi_{\mathbf{k}_{i}}(\mathbf{R})
$$

where $\psi_{i}$ is a solution of the leptonic eigenvalue equation

$$
\hat{\mathrm{H}}^{\mathrm{lep}} \psi_{i}=V_{i}^{\mathrm{lep}}(R) \psi_{i}
$$

depending parametrically on the distance $R \equiv r_{\mathrm{pp}}$. The leptonic Hamiltonian, describing the interacting hydrogenantihydrogen system, is given by

$$
\hat{\mathrm{H}}^{\mathrm{lep}}=-\frac{1}{2} \nabla_{e}^{2}-\frac{1}{2} \nabla_{\bar{e}}^{2}-\frac{1}{r_{p e}}+\frac{1}{r_{p e}^{-}}+\frac{1}{r_{p \bar{e}}}-\frac{1}{r_{p \bar{e}}^{-}}-\frac{1}{r_{e \bar{e}}} .
$$

In the present context of a collision of a hydrogen and an antihydrogen atom in their ground states, the wave function $\psi_{i}\left(R ; \mathbf{r}_{e}, \mathbf{r}_{e}^{-}\right)$is equivalent to the leptonic ground-state wave function of the $\mathrm{H}-\overline{\mathrm{H}}$ molecule that is rotationally symmetric around the interhadronic axis. This wave function describes a $\Sigma$ state with zero projection of the total leptonic angular momentum on the internuclear axis.

The hadronic wave function $\chi_{\mathbf{k}_{i}}(\mathbf{R})$ is a solution to the wave equation

$$
\left(-\frac{1}{m_{p}} \nabla_{R}^{2}+V_{i}(R)\right) \chi_{\mathbf{k}_{i}}(\mathbf{R})=E_{i} \chi_{\mathbf{k}_{i}}(\mathbf{R})
$$

describing the motion of the hadrons. The potential $V_{i}(R)$ in Eq. (13) is given by $V_{i}(R)=V_{i}^{\text {lep }}(R)-1 / R$. Therefore, in order to solve Eq. (13), it is first necessary to solve Eq. (11) on a sufficiently dense mesh of $R$ values, which yields a numerical discretized representation of $V_{i}(R)$. For $\chi_{\mathbf{k}_{i}}(\mathbf{R})$, the normalization according to

$$
\left\langle\chi_{\mathbf{k}_{i}^{\prime}} \mid \chi_{\mathbf{k}_{i}}\right\rangle=\delta\left(\epsilon_{i}-\epsilon_{i}^{\prime}\right) \delta\left(\Omega_{i}-\Omega_{i}^{\prime}\right)
$$

was adopted.

Choosing the $z$ axis as the scattering axis (i.e., $\mathbf{k}_{i}=k_{i} \hat{z}$ ), we obtain for the partial-wave expansion of $\chi_{\mathbf{k}_{i}}(\mathbf{R})$

$$
\chi_{\mathbf{k}_{i}}(\mathbf{R})=\frac{1}{R} \sum_{L_{i}} f_{L_{i}}\left(k_{i}, R\right) Y_{L_{i} 0}\left(\Omega_{R}\right),
$$

where $f_{L_{i}}\left(k_{i}, R\right) / R$ is the radial solution of Eq. (13), and $Y_{L M}$ denotes a spherical harmonic. With the normalization adopted in Eq. (14), the asymptotic form of $f_{L_{i}}$ for $R \rightarrow \infty$ is

$$
\begin{aligned}
f_{L_{i}}\left(k_{i}, R\right) \rightarrow & \frac{1}{\sqrt{2} \pi} \sqrt{\frac{\mu_{i}}{k_{i}}} \sqrt{2 L_{i}+1} i L^{L_{i}} e^{i \delta_{L_{i}}} \\
& \times \sin \left(k_{i} R-\frac{L_{i} \pi}{2}+\delta_{L_{i}}\right),
\end{aligned}
$$

where $\delta_{L_{i}}$ is the angular momentum-dependent phase shift. In the present paper, we are interested in collisions with very low kinetic energies in the initial channel, and thus, we need to take only $s$-wave scattering into account, for which 


$$
f_{0}\left(k_{i}, R\right) \rightarrow \frac{1}{\sqrt{2} \pi} \sqrt{\frac{\mu_{i}}{k_{i}}} e^{i \delta_{0}} \sin \left(k_{i} R+\delta_{0}\right) .
$$

The $s$-wave approximation is justified when $k_{i} R \ll 1$, which for $R \simeq 1$ implies $\epsilon_{i} \ll 10^{-4}$.

If we partition the Hamiltonian in the final channel and ignore the small recoil transferred to the protonium atom due to the large mass difference between protonium and positronium, the final-state wave function as an eigenfunction of $\hat{H}_{f}$, may be expressed in the form

$$
\Upsilon_{f}\left(\mathbf{R}, \mathbf{r}_{e}, \mathbf{r}_{e}^{-}\right)=\Phi_{E, \Omega_{f}}\left(\mathbf{r}_{e}, \mathbf{r}_{e}^{-}\right) \widetilde{\phi}_{N L M}(\mathbf{R})
$$

where $\widetilde{\phi}_{N L M}(\mathbf{R})$ is a hydrogenic wave function describing the bound protonium, and $\Phi_{E, \Omega_{f}}\left(\mathbf{r}_{e}, \mathbf{r}_{e}^{-}\right)$is the wave function describing a freely moving positronium in the state $n l m$ with momentum $\mathbf{k}_{f} \cdot \Phi_{E, \Omega_{f}}\left(\mathbf{r}_{e}, \mathbf{r}_{e}^{-}\right)$is given by

$$
\Phi_{E, \Omega_{f}}\left(\mathbf{r}_{e}, \mathbf{r}_{e}^{-}\right)=\frac{1}{(2 \pi)^{3 / 2}} \sqrt{\mu_{f} k_{f}} e^{i \mathbf{k}_{f} \mathbf{r}_{e e}^{\mathrm{cm}}} \phi_{n l m}\left(\mathbf{r}_{e}^{-}\right)
$$

where $\mathbf{r}_{e e^{-}}^{\mathrm{cm}}=\left(\mathbf{r}_{e}+\mathbf{r}_{e}^{-}\right) / 2$. The index $E$ of $\Phi_{E, \Omega_{f}}\left(\mathbf{r}_{e}, \mathbf{r}_{e}^{-}\right)$labels the total translational and internal energy of the positronium atom, i.e., $E=\epsilon_{f}+\mathcal{E}_{n}^{\mathrm{Ps}}$ and $\Omega_{f}$ is the direction of the final momentum.

\section{Separation of the transition matrix}

The application of the Born-Oppenheimer approximation to the initial-state wave function, together with the partitioning of the Hamiltonian in the final channel, leads to the separation of the transition matrix element $T_{f i}$, defined in Eq. (7), into a leptonic and a hadronic contribution, as is evident from rewriting $T_{f i}$ with the aid of Eqs. (10) and (18), as

$$
T_{f i}=\left\langle\Phi_{E, \Omega_{f}}\left(\mathbf{r}_{e}, \mathbf{r}_{e}^{-}\right) \widetilde{\phi}_{N L M}(\mathbf{R})\left|\hat{\mathrm{V}}_{f}\right| \psi_{i}\left(R ; \mathbf{r}_{e}, \mathbf{r}_{e}^{-}\right) \chi_{\mathbf{k}_{i}}(\mathbf{R})\right\rangle .
$$

The two contributions are coupled to each other via the parametric dependence of the leptonic wave function $\psi_{i}\left(R ; \mathbf{r}_{e}, \mathbf{r}_{e}^{-}\right)$on the interhadronic distance $R$.

Separating the (hydrogenlike) protonium wave function into a radial and an angular part,

$$
\widetilde{\phi}_{N L M}(\mathbf{R})=\frac{1}{R} \tilde{u}_{N L}(R) Y_{L M}\left(\Omega_{R}\right)
$$

and using the corresponding separation of $\chi_{\mathbf{k}_{i}}(\mathbf{R})$ [see Eq. (15)], we carry out the integration over the angular part of $\mathbf{R}$. Using the orthogonality of the spherical harmonics, we obtain

$$
T_{f i}=\delta_{L, 0} \delta_{M, 0}\left\langle\Phi_{E, \Omega_{f}}\left(\mathbf{r}_{e}, \mathbf{r}_{e}^{-}\right) \frac{\tilde{u}_{N L}(R)}{R}\left|\hat{\mathrm{V}}_{f}\right| \psi_{i}\left(R ; \mathbf{r}_{e}, \mathbf{r}_{e}^{-}\right) \frac{f_{0}\left(k_{i}, R\right)}{R}\right\rangle
$$

in the case of $s$-wave scattering.

The final calculation of $T_{f i}$ splits naturally into two parts. First, the leptonic matrix element

$$
t_{E, i}\left(R ; \Omega_{f}\right)=\left\langle\Phi_{E, \Omega_{f}}\left(\mathbf{r}_{e}, \mathbf{r}_{e}^{-}\right)\left|\hat{\mathrm{V}}_{f}\right| \psi_{i}\left(R ; \mathbf{r}_{e}, \mathbf{r}_{e}^{-}\right)\right\rangle
$$

is calculated as a function of $R$. It is convenient to expand the leptonic matrix element in the angular momentum $l_{f}, m_{f}$ of the positronium atom

$$
t_{E, i}\left(R ; \Omega_{f}\right)=\sum_{l_{f}, m_{f}} \tilde{t}_{E, i}^{l_{f}^{m_{f}}}(R) Y_{l_{f} m_{f}}\left(\Omega_{f}\right) .
$$

For molecular $\Sigma$ symmetry $m_{f}=0$. Second, the total transition matrix element is calculated by an integration over $R$ according to

$$
T_{f i}=\delta_{L, 0} \delta_{M, 0} \int_{0}^{\infty} \tilde{u}_{N 0}(R) t_{E, i}\left(R ; \Omega_{f}\right) f_{0}\left(k_{i}, R\right) d R .
$$

Hence, at low energies, protonium can only be formed in the $L=0$ state. Using Eqs. (6), (24), and (25), and doing the replacement $\Sigma_{f} \longrightarrow \Sigma_{N} \int d \Omega_{f}$, we then obtain the rearrangement cross section

$$
\sigma^{\text {rearr }}=\frac{(2 \pi)^{4}}{k_{i}^{2}} \sum_{N, l_{f}}\left|\int_{0}^{\infty} \tilde{u}_{N 0}(R) \tilde{t}_{E, i}^{l_{f}^{0}}(R) f_{0}\left(k_{i}, R\right) d R\right|^{2} .
$$

Since only a small amount of energy is released in the final channel (see Table I) the contributions from partial waves $l_{f}>0$ are negligible. Moreover, symmetry under the combination of lepton exchange and horizontal reflection [4] implies that all odd partial waves in the final channel vanish. Hence, keeping only the $s$-wave contribution is a good approximation,

$$
\sigma^{\text {rearr }}=\frac{(2 \pi)^{4}}{k_{i}^{2}} \sum_{N}\left|\int_{0}^{\infty} \tilde{u}_{N 0}(R) \tilde{t}_{E, i}^{00}(R) f_{0}\left(k_{i}, R\right) d R\right|^{2} .
$$

In the next section, we shall describe how the leptonic matrix elements were calculated.

\section{Computational details}

\section{Computation of the initial-state wave function}

In the spirit of the Born-Oppenheimer approximation that has been applied to the initial-channel wave function 
$\Psi_{\mathbf{k}_{i}}^{(+)}\left(\mathbf{R} ; \mathbf{r}_{e}, \mathbf{r}_{e}^{-}\right)$[see Eq. (10)], the calculation of $\Psi_{\mathbf{k}_{i}}^{(+)}$naturally splits into two parts. First, the leptonic wave function $\psi_{i}\left(R ; \mathbf{r}_{e}, \mathbf{r}_{e}^{-}\right)$and the corresponding leptonic energies $V_{i}^{\mathrm{lep}}(R)$ are obtained by solving Eq. (11) for a number of interhadronic distances $R$. In the present paper, Eq. (11) is solved by introducing explicitly correlated basis functions in prolate spheroidal coordinates. This approach allows a very efficient and accurate treatment of leptonic correlation and has been very successful when solving the leptonic eigenvalue problem of molecular hydrogen. Due to the introduction of a finite, but nonorthogonal basis set, Eq. (11) becomes a finite generalized matrix eigenvalue problem that can be solved by standard diagonalization techniques. The resulting leptonic wave function can be written as

$$
\begin{aligned}
\psi_{i}= & \sum_{j=1}^{N} c_{i j} e^{-\alpha\left(\xi_{e}+\xi_{e}^{-}\right)}\left\{e^{\beta\left(\eta_{e}-\eta_{e}^{-}\right)} \xi_{e}^{p_{j}} \xi_{\bar{e}}^{\bar{p}_{j}} \eta_{e}^{q_{j}} \eta_{\bar{e}}^{\bar{q}_{j}}\right. \\
& \left.+(-1)^{q_{j}+\bar{q}_{j}} e^{-\beta\left(\eta_{e}-\eta_{e}^{-}\right)} \xi_{e}^{\bar{p}_{j}} \xi_{\bar{e}}^{p_{j}} \eta_{e}^{\bar{q}_{j}} \eta_{-}^{q_{j}}\right\} \rho^{\mu_{j}},
\end{aligned}
$$

where $N$ is the dimension of the basis, $\xi_{i}, \eta_{i}$ are the prolate spheroidal coordinates of the leptons, and $\rho=2 r_{e e} / R$. The positive integers $p_{j}, \bar{p}_{j}, q_{j}, \bar{q}_{j}$, and $\mu_{j}$, as well as the nonlinear parameters $\alpha$ and $\beta$ are fixed for a specific basis, while the linear parameters $c_{i j}$ are obtained from the matrix diagonalization. Based on the variational principle, the nonlinear parameters $\alpha$ and $\beta$ have been optimized for every value of $R$ by minimizing the (leptonic) ground-state energy $V_{i}^{\mathrm{lep}}(R)$ of $\mathrm{H}-\overline{\mathrm{H}}$.

The trial function (28) is the same as the one used by Kołos et al. [4], except that we used two, rather than four, independent nonlinear parameters. Although the old basis with four nonlinear parameters seems to be superior to the present one, since it is more flexible, it turns out that restricting the number of nonlinear parameters to two, allowed us to use much larger basis sets before running into numerical problems caused by the use of basis functions that are nearly linearly dependent. In the most interesting range of $R(0.85$ $\leqslant R \leqslant 3.6$ ), it was possible to use $N=908$ basis functions. For larger values of $R$, the dimension $N$ was reduced to $N$ $=114$ at $R=13$ in a number of steps. In addition to allowing larger basis sets, the form of Eq. (28) turned out to be much less restrictive regarding the choice of the integer parameters. While the old basis usually requires a careful selection of basis functions in order to prevent numerical problems, the new basis allowed us to choose the integer parameters according to general rules. The $N=908$ basis was composed by selecting sets of integer parameters $\left\{\mu_{j}, p_{j}, \bar{p}_{j}, q_{j}, \bar{q}_{j}\right\}$ in the following way. For $\mu_{j}=0$, all sets with $p_{j}, \bar{p}_{j}, q_{j}, \bar{q}_{j} \leqslant 6$ and $p_{j}+\bar{p}_{j}, q_{j}+\bar{q}_{j} \leqslant 8$ were selected. In addition, all sets fulfilling $p_{j}, \bar{p}_{j}, q_{j}, \bar{q}_{j} \leqslant 2$ were used for $\mu_{j}=1,2$, and 3. For symmetry reasons, it is necessary to include only one of those sets that become identical, if $p_{j}$ and $\bar{p}_{j}$ as well as $q_{j}$ and $\bar{q}_{j}$ are simultaneously exchanged.

In order to assess the accuracy of the leptonic wave function, we compare in Table II our result for the ground-state potential curve $V_{i}(R)$, obtained from the leptonic energy
TABLE II. Comparison of the hydrogen-antihydrogen groundstate energies $V_{i}(R)$ in the Born-Oppenheimer approximation.

\begin{tabular}{lccc}
\hline \hline$R$ & This work & Ref. [7] & Ref. [4] \\
\hline 0.85 & -1.428121 & -1.4289 & \\
0.90 & -1.368924 & -1.3682 & \\
0.95 & -1.317803 & -1.3174 & -1.314522 \\
1.00 & -1.273695 & -1.2723 & -1.271095 \\
1.50 & -1.060987 & & -1.060540 \\
2.00 & -1.013413 & & -1.010219 \\
3.00 & -1.001344 & & -1.001280 \\
5.00 & -1.000472 & & -1.000463 \\
10.00 & -1.000008 & & -1.000008 \\
\hline \hline
\end{tabular}

$V_{i}^{\text {lep }}(R)$ by adding the interhadronic potential $-1 / R$, with that of Kołos et al. [4], which for a long time, was the most accurate potential available. Recently, it has been improved in the short $R$ range $(0.8 \leqslant R \leqslant 1.0)$ by Armour et al. [7] who included the channel describing positronium formation explicitly in their basis. The authors claimed that this was crucial in order to be able to improve on the potential given in Ref. [4], and indeed it seems to be effective for $R<0.9$. However, our ground-state potential is more accurate than the ones previously available, including the one of Armour et al., for $R \geqslant 0.9$, although our approach does not include the rearrangement channel explicitly. The correlation of the leptonic pair is, however, present in our basis functions due to the explicit occurrence of the interleptonic distance $r_{e \bar{e}}$.

The potential $V_{i}(R)$ that governs the motion of the hadrons in the initial channel of the collision, is presented in Fig. 2. As is seen from the figure, the ground state is purely attractive, i.e., there are no potential wells or barriers that could temporarily trap the atom-antiatom system, preventing the hadrons from coming close to each other and, ultimately, annihilate. Armour and Zeman have calculated the first-order corrections to the Born-Oppenheimer approximation to $V_{i}(R)$, and found this correction to be small [14]. This indi-

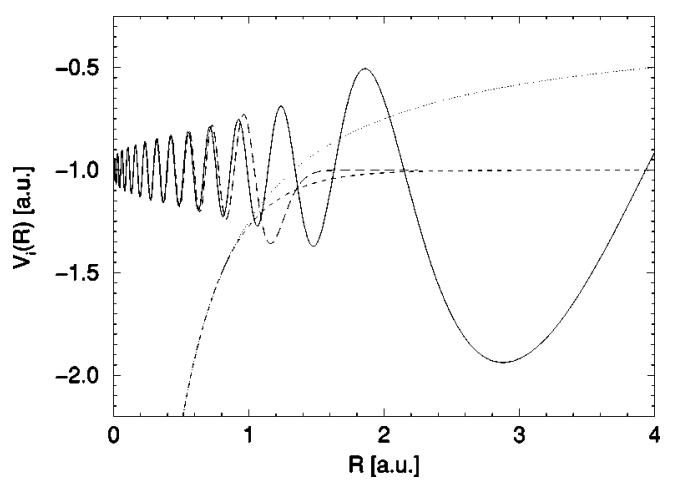

FIG. 2. The hadronic wave functions superimposed on the potential $V_{i}(R)$ formed by the leptons and the interhadronic attraction. Full line: the radial part of the hadronic scattering wave function $f_{0}\left(k_{i}, R\right)$ for $\epsilon_{i}=10^{-10}$; Long dashed: the radial part $\tilde{u}_{24,0}(R)$ of the final Pn state; dashed: ground-state interaction potential $V_{i}(R)$; dotted: (for comparison) the proton-antiproton attraction (shifted by $\left.\mathcal{E}_{1}^{\mathrm{ps}}=-0.25\right)$. 
cates that the approach we adopted of calculating the initialchannel wave function within the Born-Oppenheimer approximation, is justified.

For small $R$ values, the leptonic potential $V_{i}^{\text {lep }}(R)$ approaches from below its limiting values $V_{i}^{\text {lep }}(0)=-0.25$, equal to the positronium binding energy. However, this limiting value is reached already at a finite distance $R_{c}=0.8$ [7], reflecting the fact that, in the adiabatic approach, the hadronic dipole potential ceases to bind the leptons below a certain critical separation $R_{c}$. In this respect, the situation resembles the three-body case, where it has been shown that a proton-antiproton pair cannot bind an electron below a critical separation $R_{c}=0.64$ [15]. In view of these findings, we construct the leptonic potential $V_{i}^{\text {lep }}(R)$ by making a smooth interpolation between our numerical value at $R$ $=0.85$ and the energy -0.25 , which is the lowest eigenvalue of Eq. (11) for $R \leqslant 0.8$.

The calculation of the hadronic scattering wave function requires, especially for low collisional energies, an accurate leptonic potential for large values of $R$. It is very difficult to calculate long-range potentials by variational methods. However, asymptotic forms can be used. In the present case, the effective asymptotic interaction potential has the form $\lim _{R \rightarrow \infty} V_{i}^{\mathrm{lep}}(R)=C_{6} / R^{6}+C_{8} / R^{8}$. The constants $C_{6}$ $=-6.499$ and $C_{8}=-124.399$ are the same as for the $\mathrm{H}_{2}$ molecule [16]. This asymptotic form was found to deviate by only $3 \times 10^{-8}$ from our calculated energy at $R=13$. This indicates the high accuracy of our potential and allows its continuation beyond $R=13$.

After having obtained the potential $V_{i}(R)$, the radial part of the hadronic wave-function $\chi_{\mathbf{k}_{i}}(\mathbf{R})$, i.e., $f_{0}\left(k_{i}, R\right)$, was obtained by numerical integration of Eq. (13), starting from $R=0$. The integration was continued up to a sufficiently large value of $R$, at which the wave function takes to within seven significant digits, the asymptotic behavior expected from Eq. (17). The asymptotic amplitude then fixes the normalization of the radial solutions $f_{0}\left(k_{i}, R\right)$. At $R \rightarrow 0$, $f_{0}\left(k_{i}, R\right)$ was matched to a Coulomb wave. The resulting radial wave function $f_{0}\left(k_{i}, R\right)$ is presented in Fig. 2, together with the radial part $\tilde{u}_{N 0}(R)$ of the hadronic wave function in the final channel [cf. Eq. (21)] that describes the excited $(N=24)$ bound state of protonium.

\section{Computation of the final-state wave function}

The final-channel wave function $\Upsilon_{f}\left(\mathbf{R}, \mathbf{r}_{e}, \mathbf{r}_{e}^{-}\right)$is a freewave solution to Eq. (8) describing the positroniumprotonium pair moving freely with the relative energy $\epsilon_{f}$. It can be written in the product form given by Eq. (18). Ignoring the small recoil and relativistic corrections, the hadronic part is given analytically by the wave function describing the excited state of protonium, $\widetilde{\phi}_{N L M}(\mathbf{R})$. Ignoring relativistic effects, also the leptonic wave function $\Phi_{E, \Omega_{f}}\left(\mathbf{r}_{e}, \mathbf{r}_{e}^{-}\right)$[see Eq. (19)] is known analytically, since it describes a positronium atom moving with respect to protonium with the energy $\epsilon_{f}=k_{f}^{2} /\left(2 \mu_{f}\right)$.

However, using the numerical form (28) of the initialstate wave function, the calculation of the leptonic transition
TABLE III. Convergence of the positronium ground-state energy, its error relative to the exact ground-state energy -0.25 , and the convergence of the squared leptonic matrix element (30) with increasing $\mu_{\max }$ used in the basis set [cf. Eq. (28)] for $R=1.0$.

\begin{tabular}{lccc}
\hline \hline$\mu_{\max }$ & $\mathcal{E}_{n}^{\text {Ps }}$ & Rel. error $[\%]$ & $\left|\tilde{t}_{E, i}(1)\right|^{2}$ \\
\hline 0 & -0.1376 & 45.0 & 0.0722 \\
1 & -0.1913 & 23.5 & 0.0573 \\
2 & -0.2193 & 12.3 & 0.0530 \\
3 & -0.2348 & 6.1 & 0.0494 \\
\hline \hline
\end{tabular}

matrix element $t_{E, i}\left(R ; \Omega_{f}\right)$ is a formidable task if the analytic form (19) of the final leptonic wave function is adopted. In order to perform the integration, both wave functions (in the initial and final channels) have to be expressed in a common coordinate system. Unfortunately, the final wave function cannot be easily expressed in the prolate spheroidal coordinate system, nor can the initial-state wave function easily be transformed into a coordinate system that allows a simple description of the final-state wave function.

In order to facilitate the calculation of $t_{E, i}\left(R ; \Omega_{f}\right)$ we also expanded the leptonic wave function $\Phi_{E, \Omega_{f}}\left(\mathbf{r}_{e}, \mathbf{r}_{e}^{-}\right)$of the final channel in prolate-spheroidal coordinates. For this purpose, the leptonic final-channel Hamiltonian that describes a freely moving positronium atom

$$
\hat{H}_{f}^{\mathrm{lep}}=\hat{H}^{\mathrm{Ps}}=-\frac{1}{2} \nabla_{e}^{2}-\frac{1}{2} \nabla_{\bar{e}}^{2}-\frac{1}{r_{e \bar{e}}},
$$

has been diagonalized in the same basis Eq. (28) that was used for diagonalizing the leptonic part of the total Hamiltonian $\hat{H}^{\text {lep }}$. One can easily obtain $\hat{H}^{\mathrm{Ps}}$ from $\hat{H}^{\text {lep }}$ by removing the terms that describe hadron-lepton interactions. It still contains the center-of-mass motion, since the kinetic-energy operators for both leptons are included.

The present approach leads to a discretization of the continuous spectrum of the moving positronium, resulting in $\mathcal{L}^{2}$ eigensolutions $\Phi_{E_{j}, \Omega_{f}}$ corresponding to a discretized set of eigenvalues $E_{j}$. The following section describes how these discretized solutions have been used in the calculation of the leptonic transition matrix elements. Before that, we comment on the validity and accuracy of this approximation. The approach is formally correct and would yield the exact leptonic wave function in the limit of an infinite basis set, since the adopted basis is in principle flexible enough. In practice, however, the limitation to $\mu_{j} \leqslant 3$ and the requirement of using a finite basis leads to a loss in accuracy. The convergence of the positronium ground-state energy $\mathcal{E}_{n}^{\text {Ps }}$ with increasing maximum value $\mu_{\max }$ of $\mu_{j}$, is displayed in Table III. We find that the relative error in $\mathcal{E}_{n}^{\mathrm{Ps}}$ is approximately halved each time $\mu_{\max }$ is increased one step. In addition, there is a small artificial $R$ dependence of about \pm 0.005 .

The basis functions Eq. (28) are not adapted to the rotational symmetry of Hamiltonian $\hat{\mathrm{H}}^{\mathrm{Ps}}$ in Eq. (29). Hence, the discretized eigensolutions obtained cannot be classified according to a particular direction of the final momentum, as 
the wave-function $\Phi_{E, \Omega_{f}}$ in Eq. (19) are, and neither according to a particular final angular momentum $l_{f}$. The use of ansatz Eq. (28) for the wave function will therefore by necessity yield the leptonic matrix element summed over all directions of the final momentum $\Omega_{f}$, or, equivalently, over all final angular momenta $l_{f}$. As discussed above in Sec. II B 3, it is, however, a good approximation to represent this sum over all angular momenta by the $l_{f}=0$ term only.

There is another possible problematic limitation when expressing the wave function of the moving positronium by a wave function of the type given in Eq. (28), since this wave function is by construction rotationally symmetric around the interhadronic axis, i.e., it has $\Sigma$-type symmetry. A positronium atom that moves with respect to the center-of-mass of the protonium (i.e., with respect to the center of the interhadronic axis) will, however, not be restricted to that symmetry. Nevertheless, since there is no term in the Hamiltonian $\hat{\mathrm{H}}^{\mathrm{Ps}}$ in Eq. (29) that couples states with different projections along $\mathbf{R}$ of the total leptonic angular momentum $\Lambda$ $=\Sigma, \quad \Pi$, etc., it is possible to evaluate the solutions with different values of $\Lambda$ independently of each other. Because the initial-state wave function and the interaction potential $\hat{\mathrm{V}}_{f}$ are rotationally symmetric around the interhadronic axis, only final-state wave functions with the same rotational symmetry will give nonzero contributions to the transition matrix element. Therefore, the symmetry-restricted ansatz for the wave function of the moving positronium and the limitation to $\Sigma$-type symmetry are appropriate.

\section{Calculation of the leptonic matrix element}

After the leptonic initial- and final-channel wave functions have been obtained, the leptonic transition matrix element can be evaluated. For a selected initial collision energy $\epsilon_{i}$ and final protonium state $N$ the energy $E$ at which $\widetilde{t}_{E, i}^{00}(R)$ has to be calculated, follows directly from the energy conservation as $E=E_{i}-\mathcal{E}_{N}^{\mathrm{Pn}}=\epsilon_{i}-1-\mathcal{E}_{N}^{\mathrm{Pn}}$.

Obviously, it is unlikely to get from the diagonalization of $\hat{\mathrm{H}}_{f}^{\text {lep }}$ [Eq. (29)], in a discrete basis, the solutions at the particular energy $E_{j}=E$; besides, the discretized solutions, being square integrable, are not properly normalized. To obtain the transition matrix element $\widetilde{t}_{E, i}^{00}(R)$ we apply the method based on the use of complex coordinates as in Ref. [17]. The application of this approach to the present problem will be briefly described.

The idea is to extract the transition matrix element as the imaginary part of the expectation value of the operator $\hat{\mathrm{V}}_{f} \hat{G}_{f}^{\text {lep }} \hat{\mathrm{V}}_{f}$ when applied to the leptonic ground state in the initial channel

$$
\begin{aligned}
& \left|\tilde{t}_{E, i}(R)\right|^{2} \equiv \sum_{l_{f}}\left|\tilde{t}_{E, i}^{l_{f}^{0}}(R)\right|^{2}=\int d \Omega_{f}\left|t_{E, i}\left(R ; \Omega_{f}\right)\right|^{2} \\
& =\frac{1}{\pi} \operatorname{Im}\left\{\left\langle\psi_{i}\left|\hat{\mathrm{V}}_{f} \hat{G}_{f}^{\mathrm{lep}}(E) \hat{\mathrm{V}}_{f}\right| \psi_{i}\right\rangle\right\},
\end{aligned}
$$

where $\hat{\mathrm{G}}_{f}^{\mathrm{lep}}(E)$ is the resolvent operator of the leptonic finalchannel Hamiltonian

$$
\hat{\mathrm{G}}_{f}^{\mathrm{lep}}(E)=\lim _{\epsilon \rightarrow 0}\left(\hat{H}_{f}^{\mathrm{lep}}-E-i \varepsilon\right)^{-1} .
$$

Introducing the complete eigenstate expansion of the operator $\hat{\mathrm{H}}_{f}^{\text {lep }}$, we obtain

$$
\begin{aligned}
& \left|\tilde{t}_{E, i}(R)\right|^{2} \\
& \quad=\frac{1}{\pi} \operatorname{Im}\left\{\lim \sum \int \frac{\left\langle\psi_{i}\left|\hat{\mathrm{V}}_{f}\right| \Phi_{E_{j}, \Omega_{f}}\right\rangle\left\langle\Phi_{E_{j}, \Omega_{f}}\left|\hat{\mathrm{V}}_{f}\right| \psi_{i}\right\rangle}{E_{j}-E-i \varepsilon}\right\} \\
& \quad=\int d \Omega_{f}\left\langle\psi_{i}\left|\hat{\mathrm{V}}_{f}\right| \Phi_{E, \Omega_{f}}\right\rangle\left\langle\Phi_{E, \Omega_{f}}\left|\hat{\mathrm{V}}_{f}\right| \psi_{i}\right\rangle \\
& \quad=\left.\int d \Omega_{f}\left\langle\Phi_{E, \Omega_{f}}\left|\hat{\mathrm{V}}_{f}\right| \psi_{i}\right\rangle\right|^{2}
\end{aligned}
$$

where the symbol $\Sigma \int$ denotes the summation over the discrete part and integration over the continuous part of the spectrum of $\hat{\mathrm{H}}_{f}^{\text {lep }}$, and summation over all other quantum numbers specifying the final state, including the direction of the final momentum. In the present case, only the continuous spectrum is present, as $\hat{\mathrm{H}}_{f}^{\text {lep }}$ describes the moving positronium.

The transition matrix element $\left|\widetilde{t}_{E, i}(R)\right|^{2}$ can be obtained as the ground-state expectation value given in Eq. (30), provided one can find a meaningful numerical evaluation of the resolvent operator. This is done with the help of the complex-coordinate method.

The original Hamiltonian is transformed by the dilation operation

$$
\hat{\mathrm{H}} \rightarrow \hat{\mathrm{H}}^{\theta} \equiv \hat{\mathrm{U}}(\theta) \hat{\mathrm{H}} \hat{\mathrm{U}}^{-1}(\theta) ; \hat{\mathrm{U}}(\theta) f(\mathbf{r})=e^{3 \theta / 2} f\left(e^{\theta} \mathbf{r}\right),
$$

where $\theta$ is the complex dilation parameter.

The leptonic transition matrix element in Eq. (23) is invariant under the dilation transformation

$$
\begin{aligned}
t_{E, i}\left(R ; \Omega_{f}\right) & =\left\langle\left(\hat{\mathrm{U}}^{\dagger}\right)^{-1}(\theta) \Phi_{E_{j}, \Omega_{f}}\left|\hat{\mathrm{U}}(\theta) \hat{\mathrm{V}}_{f} \hat{\mathrm{U}}^{-1}(\theta)\right| \hat{\mathrm{U}}(\theta) \psi_{i}\right\rangle \\
& =\left\langle\Phi_{\mathcal{E}_{j}, \Omega_{f}}^{\theta^{*}}\left|\hat{\mathrm{V}}_{f}^{\theta}\right| \psi_{i}^{\theta}\right\rangle .
\end{aligned}
$$

The discretized approximation to such an analytically continued transition matrix element does not vanish, and can be obtained with the help of the biorthonormal expansion in terms of square-integrable solutions $\Phi_{\mathcal{E}_{j}, \Omega_{f}}^{\theta}, \Phi_{\mathcal{E}_{j}, \Omega_{f}}^{\theta^{*}}$, corresponding to complex eigenvalues $\mathcal{E}_{j}$, obtained by diagonalizing the complex-dilated Hamilton operator $\hat{H}_{f}^{\text {lep, } \theta}$.

The transition matrix element $\widetilde{t}_{E, i}(R)$ can then be obtained from the dilated expression introduced in Eq. (32), i.e., from 


$$
\left|\tilde{t}_{E, i}(R)\right|^{2}=\int d \Omega_{f}\left|\left\langle\Phi_{E, \Omega_{f}}\left|\hat{\mathrm{V}}_{f}\right| \psi_{i}\right\rangle\right|^{2}=\frac{1}{\pi} \operatorname{Im}\left\{\sum_{j} \int d \Omega_{f} \frac{\left\langle\psi_{i}^{\theta *}\left|\hat{\mathrm{V}}_{f}^{\theta}\right| \Phi_{\mathcal{E}_{j}, \Omega_{f}}^{\theta}\right\rangle\left\langle\Phi_{\mathcal{E}_{j}, \Omega_{f}}^{\theta *}\left|\hat{\mathrm{V}}_{f}^{\theta}\right| \psi_{i}^{\theta}\right\rangle}{\mathcal{E}_{j}-E}\right\},
$$

which does not contain the limiting procedure with respect to $\varepsilon$ and converges to the proper limit owing to the analyticity retained through complex scaling. Evidently, it is necessary to apply the complex-dilation transformation also to the total leptonic Hamiltonian $\hat{\mathrm{H}}^{\text {lep }}$ in order to obtain $\psi_{i}^{\theta}$ and $\psi_{i}^{\theta *}$.

An example of the convergence of $\left|\tilde{t}_{E, i}(R)\right|^{2}$ with respect to the maximum value of $\mu_{i}$ used in Eq. (28) is displayed in Table III. We find that the trend of the convergence is similar to that of the positronium ground-state energy. Based on the known $6 \%$ relative error of the best value for the positronium ground state, we then estimate the error in $\left|\tilde{t}_{E, i}(R)\right|^{2}$ due to basis-set inaccuracies to be less than $25 \%$.

The calculation according to Eq. (35) gives the absolute value squared of the leptonic matrix element summed over partial waves. Hence, we cannot separate the different partial waves, as needed in Eq. (26). However, since we may neglect higher final partial waves we have $\widetilde{t}_{E, i} \simeq \widetilde{t}_{E, i}^{00}$, which gives the leptonic matrix element needed in the final $s$-wave approximation Eq. (27).

\section{Results for the rearrangement cross section}

With the aid of the hadronic wave functions of the initial and final channel and the leptonic transition matrix element, it is finally possible to evaluate the total transition matrix element $T_{f i}$ with the aid of Eq. (25), and to obtain the cross section $\sigma^{\text {rearr }}$ for rearrangement using Eq. (6). The integrand in Eq. (25) involves two rapidly oscillating functions, the highly excited protonium state, and the low-energy hydrogen-antihydrogen wave function (see Fig. 2). At very short distances, both systems interact with an $R^{-1}$ potential, and hence, oscillate in phase, while the last lobe of the bound-state wave function is $180^{\circ}$ out of phase with the continuum state. The leptonic matrix element $\widetilde{t}_{E, i}^{00}(R)$, which varies slowly with $R$, provides a weighting of the positive and negative contributions to the overlap of the hadronic wave functions. This situation gives rise to a cancellation effect in the integral Eq. (25), which makes the result very sensitive to small changes in $f_{0}\left(k_{i}, R\right)$. On the other hand, $\widetilde{t}_{E, i}^{00}(R)$ vanishes as $R \rightarrow 0$ because $\hat{\mathrm{V}}_{f}$ vanishes in this limit. This limits the effect of possible inaccuracies in $f_{0}\left(k_{i}, R\right)$ at small internuclear distances. We believe the molecular data used to calculate $f_{0}\left(k_{i}, R\right)$ to be very accurate down to $R$ $=0.85$. The smooth interpolation between $R=0.80$ and $R$ $=0.85$ can be done in several different ways, but the details of this interpolation were found to have a relatively small influence on the resulting cross section, changing the result by $\sim 5 \%$.

Our results for the rearrangement cross section are presented in Fig. 3. As required, the rearrangement cross section follows Wigner's threshold law characterized by an $\epsilon_{i}^{-1 / 2}$ energy dependence, assuming for the low energies the form $\sigma^{\text {rearr }}=0.09 \epsilon_{i}^{-1 / 2}$.

In principle, there are many possible rearrangement channels determined by different principal quantum numbers $n$ $\geqslant 1$ of the positronium, and $N \leqslant 24$ of the protonium, as discussed above in Sec. II A. The rearrangement process is, however, dominated by the maximally excited final protonium state, i.e., $N=24$, and hence, $n=1$. To estimate the cross section for rearrangement into more tightly bound protonium states, we evaluated Eq. (6) with $\widetilde{t}_{E, i}^{00}(R)=1$ for different protonium states. The resulting squared overlap of the initial and final hadronic wave functions was found to be 250 times smaller for $N=23$ than for $N=24$. We therefore found it sufficient to include only the $N=24, n=1$ final state in $\sigma^{\text {rearr }}$.

In Fig. 3, we also compare our result for the rearrangement cross section to earlier results by Kołos et al. [4], and by Voronin and Carbonell [18]. The former calculation used a Born-Oppenheimer potential obtained in a way similar to ours, but used for the cross section a semiclassical estimate based on the conservation of energy and angular momentum. Voronin and Carbonell made an estimate of $\sigma^{\text {rearr }}$ based on a calculation of hydrogen-antiproton scattering, but with the correct long-range hydrogen-antihydrogen potential included. We find that our results are significantly smaller than those in the earlier works. The semiclassical result of Kołos et al., includes all partial waves, while the other two results are for $s$ waves only, but higher partial waves are not sufficient to explain the difference between the results. Also, in the case of hydrogen-antiproton scattering, the semiclassical method has been found to give too large a value for the rearrangement cross section [18].

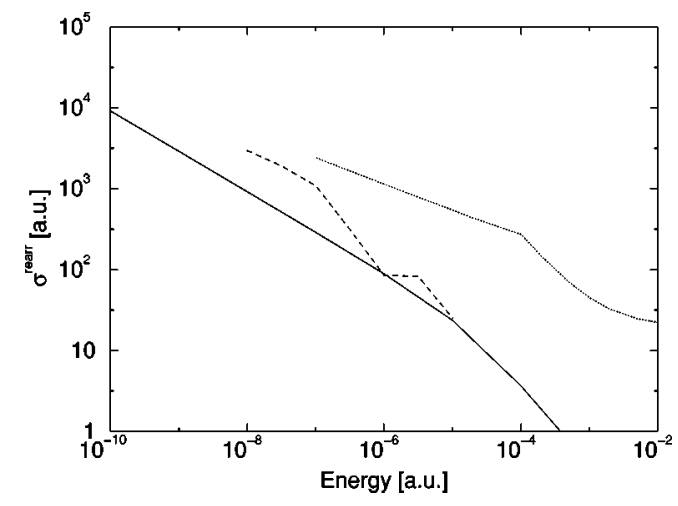

FIG. 3. Comparison of the cross section for the rearrangement calculated in this paper (full line) to a previous semiclassical calculation by Kołos et al. [4] (dotted line) and an estimate by Voronin and Carbonell [18] (dashed line). The result by Kołos et al., includes all partial waves, while the other two comprise only the $s$-wave contribution. The results from other works have been adapted from graphs. 
Kołos et al. [4] estimated the rearrangement cross section using the concept of a critical distance $R_{c}$ at which the leptons become no longer bound to the hadrons. Variational calculations show that this occurs at a value of $R$ less than $0.8[4,7]$. In the semiclassical model the rearrangement is assumed to occur spontaneously with a probability of unity on all those particle trajectories that have classical distances of closest approach less than or equal to $R_{c}$.

Our quantum-mechanical calculations do not support this model, which does not take into account the nature of the final state. The reaction can occur at zero initial kinetic energy, even at internuclear separations exceeding $R_{c}$. With the escape of the leptons from the hadrons, the energy required for reaction will be provided by the binding energy of the bound states of protonium formed by the recombination of the proton and antiproton. We have shown that the preferential channel has principal quantum number $N=24$. It is the channel for which the initial and final wave numbers are most nearly equal. A critical distance is more appropriately determined by the extent of the overlap of the initial and final-state wave functions, which Fig. 2 indicates is $R=1.3$. In any case, the calculated matrix elements yield a probability much less than unity so that a classical model will overestimate the rearrangement cross sections. A classical picture is in any case inadequate for the description of scattering in which only $s$ waves participate.

\section{ANNIHILATION IN FLIGHT}

\section{A. Proton-antiproton annihilation}

In addition to the loss of antihydrogen caused by the rearrangement collisions, there will also be loss via protonantiproton annihilation in flight according to

$$
\mathrm{H}+\overline{\mathrm{H}} \rightarrow e^{+}+e^{-}+\text {decay products, }
$$

i.e., without the formation of an intermediate protonium state.

We assume that the proton-antiproton annihilation occurs only at the exact point of coalescence of the two particles. The annihilation process may then be calculated using the pseudopotential

$$
V_{a}^{p \bar{p}}=A^{p \bar{p}} \delta(\mathbf{R}),
$$

where the annihilation constant $A^{p \bar{p}}$ can be determined from experimental data, e.g., from the width of bound protonium states. Using the pseudopotential, we may express the width of the $1 s$ protonium state in the form

$$
\Gamma_{1 s}=A^{p \bar{p}}\left|\widetilde{\phi}_{100}(0)\right|^{2}
$$

The constant $A^{p \bar{p}}$ may be calculated from Eq. (38), using the experimental value $\Gamma_{1 s}=1130 \mathrm{eV}$ from Ref. [19], obtained from studying the cascade of protonium formed after stopping antiprotons in hydrogen,

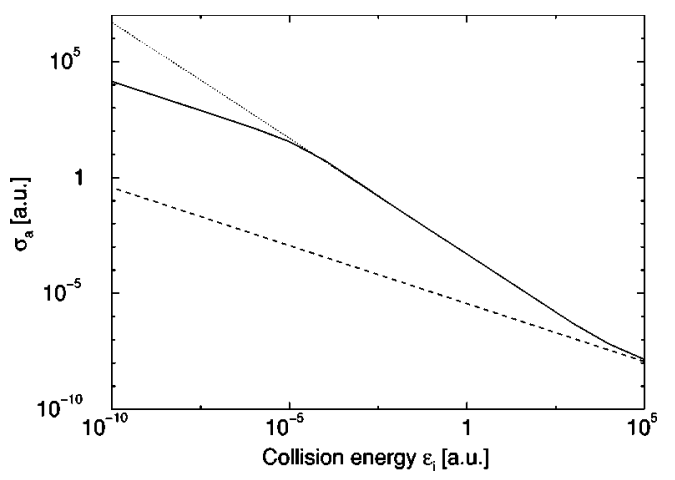

FIG. 4. The cross section for annihilation in flight, obtained according to Eq. (41) with the correct scattering wave functions (full line), and for comparison with plane waves (dashed line), and with Coulomb wave functions (dotted line). The graph has been extended to very high energies only to illustrate that the cross sections ultimately converge. The low-energy behavior of the annihilation cross sections are $0.36 \times 10^{-5} / \sqrt{\epsilon_{i}}$ (plane wave), $0.14 / \sqrt{\epsilon_{i}}(\mathrm{H}-\overline{\mathrm{H}})$, and $4.9 \times 10^{-4} / \epsilon_{i}$ (Coulomb wave).

$$
\begin{aligned}
A^{p \bar{p}} & =\frac{\Gamma_{1 s}}{\left|\widetilde{\phi}_{100}(0)\right|^{2}}=\frac{8 \pi \Gamma_{1 s}}{m_{p}^{3}}=1.69 \times 10^{-7} \\
& =6.80 \times 10^{-37} \mathrm{eV} \mathrm{m}^{3} .
\end{aligned}
$$
by

In the same way the rate for annihilation in flight is given

$$
\lambda_{a}^{p \bar{p}}=\left\langle\chi_{\mathbf{k}_{i}}(\mathbf{R})\left|V_{a}^{p \bar{p}}\right| \chi_{\mathbf{k}_{i}}(\mathbf{R})\right\rangle=A^{p \bar{p}}\left|\chi_{\mathbf{k}_{i}}(0)\right|^{2},
$$

where $\chi_{\mathbf{k}_{i}}(\mathbf{R})$ is the scattering wave function for the protonantiproton pair in the leptonic potential, defined in Eq. (13). Since all partial waves of $\chi_{\mathbf{k}_{i}}$ [see Eq. (15)] except $L_{i}=0$, vanish at the origin, only $s$ waves contribute. The annihilation rate $\lambda_{a}^{p \bar{p}}$ in Eq. (40) is given per unit energy, and depends on the incoming particle flux $F$ through the normalization of the scattering wave function $\chi_{\mathbf{k}_{i}}$. Such normalization dependence is avoided, if we instead consider the cross section. With the normalization adopted in Eq. (14), one has

$$
\sigma_{a}^{p \bar{p}}=\frac{\lambda_{a}^{p \bar{p}}}{F}=\frac{(2 \pi)^{3}}{k_{i}^{2}} A^{p \bar{p}}\left|\chi_{\mathbf{k}_{i}}(0)\right|^{2} .
$$

Here we have used the initial-state wave function $\chi_{\mathbf{k}_{i}}$ calculated without including $V_{a}^{p \bar{p}}$ in the wave equation (13).

The resulting cross section is presented in Fig. 4. To illustrate the asymptotic behavior of the cross section in the highand low-energy limits, we also present, in the same figure, the cross sections obtained for a plane wave, and for a pure Coulomb interaction characteristic of scattering of a bare proton and antiproton, respectively. The former situation corresponds to full screening of oncoming hadrons, the latter to no screening. The exact result must fall in between these two limits.

We see that for high incoming energies $\epsilon_{i}>10^{4}$, the cross sections indeed converge to the asymptotic form characteris- 


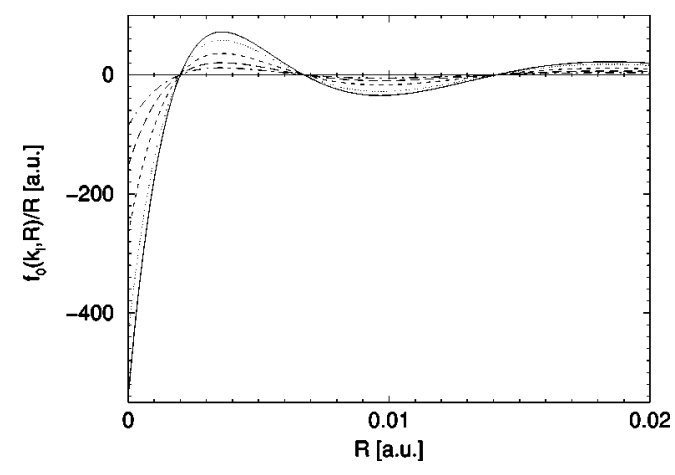

FIG. 5. The behavior of the hadronic scattering wave-function $f_{0}\left(k_{i}, R\right) / R$ at the origin. Collision energies $\epsilon_{i}=10^{-4}$ (solid), $10^{-5}$ (dotted), $10^{-6}$ (dashed), $10^{-7}$ (long dashed), and $10^{-8}$ (dotdashed).

tic of the plane-wave description. In this region, the influence of the scattering potential on the cross section is negligible. The outcome of the scattering is determined by the large collision energy rather then by the interaction potential.

In the energy region $10^{-5}-10^{4}$, the hydrogenantihydrogen cross section is almost identical to the cross section for a bare proton and antiproton. This means that the atoms feel the deeper part of the interaction potential, i.e., the short-range part, where it is completely dominated by the proton-antiproton attraction. The relatively flat long-range part of the potential, on the other hand, gives a negligible perturbation at energies in this range.

For energies smaller then $\epsilon_{i}=10^{-5}$, the $\mathrm{H}-\overline{\mathrm{H}}$ annihilation cross section deviates from the annihilation cross section for pure Coulomb attraction, and displays the $1 / \sqrt{\epsilon_{i}}$ dependence characteristic of the Wigner threshold law. The Wigner law is valid only if the potential vanishes faster than $1 / R^{2}[13]$. This indicates that it is the effective long-range interaction, rather than the Coulomb attraction, which dominates the scattering, and that the molecular or screening effects come into play. The limiting energy $10^{-5}$ for the applicability of the Wigner law coincides approximately with the binding energy of the most loosely bound state, as will be discussed in Sec. IV. Below this energy, the scattering properties are not only determined by the short-range proton-antiproton interaction, but the atoms feel the full interaction potential. This conclusion is corroborated by studying the behavior of the scattering function $\chi_{\mathbf{k}_{i}}(R)$ at the origin (see Fig. 5). The wave function shows nodes independent of the collision energy, which establishes that it is factorizable into the form $\chi_{\mathbf{k}_{i}}(R) \simeq \sqrt{\gamma\left(k_{i}\right)} \chi_{0}(R)$, where $\chi_{0}(R)$ is energy independent and the function $\gamma\left(k_{i}\right)$ can be related to the scattering amplitude. Such behavior is characteristic of low-energy scattering in the presence of a shallow bound level [20].

\section{B. Electron-positron annihilation}

It is also possible that the electron and positron will annihilate during the collision. To estimate this annihilation rate, the treatment used in the previous section for the hadrons has to be modified because ground-state positronium has two very different lifetimes depending on its spin state. For
$\operatorname{Ps}(1 s)$ in the triplet ${ }^{3} S_{1}$ state, the lifetime is $1.4 \times 10^{-7} \mathrm{~s}$, while for the singlet ${ }^{1} S_{0}$ state the lifetime is $1.25 \times 10^{-10} \mathrm{~s}$. The origin of this difference is the charge-conjugation invariance of the dominating electromagnetic decay into photons. The ${ }^{1} S_{0}$ state is even under the charge-conjugation operation $C$, and hence it can decay into two photons, each having an intrinsic charge-conjugation parity -1 , while the ${ }^{3} S_{1}$ state is odd under the same operation, and hence, must decay into three photons, which is a much slower process.

In order to accommodate both types of decays, we define two annihilation constants $A_{+}^{e \bar{e}}=4.9 \times 10^{-6}=2.0$ $\times 10^{-35} \mathrm{eV} \mathrm{m}^{3}$ for the $C$-even states, and $A_{-}^{e \bar{e}}=4.3 \times 10^{-9}$ $=1.7 \times 10^{-39} \mathrm{eV} \mathrm{m}^{3}$ for $C$-odd states. The pseudopotential for electron-positron annihilation may then be constructed by separately projecting out the even and odd parities

$$
\begin{aligned}
V_{a}^{e \bar{e}}\left(\mathbf{r}_{e e}\right)= & \frac{1}{2}(1+C) A_{+}^{e \bar{e}} \delta\left(\mathbf{r}_{e \bar{e}}\right)(1+C) \\
& +\frac{1}{2}(1-C) A_{-}^{e \bar{e}} \delta\left(\mathbf{r}_{e \bar{e}}\right)(1-C) \\
= & \frac{1}{2}\left(1-P_{e e}\right) A_{+}^{e \bar{e}} \delta\left(\mathbf{r}_{e \bar{e}}\right)\left(1-P_{e \bar{e}}\right) \\
& +\frac{1}{2}\left(1+P_{e \bar{e}}\right) A_{-}^{e \bar{e}} \delta\left(\mathbf{r}_{e \bar{e}}\right)\left(1+P_{e \bar{e}}\right) .
\end{aligned}
$$

Here, $P_{e \bar{e}}$ is the electron-positron exchange operator, $P_{e \bar{e}}$ $=-C$, where the minus sign comes from the anticommutativity of the fermionic field operators.

The hydrogen-antihydrogen system is invariant under charge conjugation of all four particles, but it is not invariant under charge conjugation of the leptons only. Expressing the initial-state wave function in terms of the coordinates $\mathbf{r}_{e \bar{e}}$ $=\mathbf{r}_{\bar{e}}-\mathbf{r}_{e}, \mathbf{r}_{\bar{e}}^{\mathrm{cm}}=\left(\mathbf{r}_{e}+\mathbf{r}_{\bar{e}}\right) / 2$, and $\mathbf{R}$, it may be expanded in partial waves even or odd under exchange of the leptons $\left[P_{e \bar{e}} Y_{l m}\left(\Omega_{r_{e \bar{e}}}\right)=(-1)^{l} Y_{l m}\left(\Omega_{r_{e \bar{e}}}\right)\right]$ as

$$
\Psi_{\mathbf{k}_{i}}^{(+)}\left(\mathbf{R}, \mathbf{r}_{e \bar{e}}^{\mathrm{cm}}, \mathbf{r}_{e \bar{e}}\right)=\sum_{l m} \Theta_{l m}\left(\mathbf{R}, \mathbf{r}_{e \bar{e}}^{\mathrm{cm}}, r_{e \bar{e}}\right) Y_{l m}\left(\Omega_{r_{e \bar{e}}}\right)
$$

In order to be single valued, $\Theta_{l m}\left(\mathbf{R}, \mathbf{r}_{e \bar{e}}^{\mathrm{cm}}, 0\right)$ can be nonvanishing only for $l=0$. Hence, we conclude that, in the contactinteraction approximation, only the even $l=0$ partial wave contributes to electron-positron annihilation. Taking also the symmetry under exchange of the leptonic spins $\chi_{S, M_{S}}$ into account we find that

$$
\begin{gathered}
\delta\left(\mathbf{r}_{e \bar{e}}\right)\left(1-P_{e \bar{e}}\right) \Psi_{\mathbf{k}_{i}}^{(+)} \chi_{1 M_{S}}=0 \\
\delta\left(\mathbf{r}_{e \bar{e}}\right)\left(1+P_{e \bar{e}}\right) \Psi_{\mathbf{k}_{i}}^{(+)} \chi_{00}=0 .
\end{gathered}
$$

Hence, even though the hydrogen-antihydrogen system is not an eigenstate with respect to charge conjugation of the leptons, the same selection rules as for the positronium ground 
state applies, i.e., two-photon decays for the singlet state, and three-photon decays for the triplet states.

The ratio of triplet collisions to singlet collisions will depend on the experimental conditions. For a statistical mixture, the ratio is 3 to 1 . In a magnetic trap, the electron spin will be parallel to the magnetic field, while the positron spin, being parallel to the magnetic moment, will point in the opposite direction. Hence, there will be singlet and triplet collisions in equal proportions. We note that the annihilation constant $A_{+}^{e \bar{e}}$ for the singlet state is larger than that for proton-antiproton annihilation. On the other hand, as is evident from Fig. 5, the proton-antiproton probability density is drastically enhanced at $R=0$, where the proton-antiproton annihilation takes place, while the leptonic annihilation occurs at a typical internuclear separation $R \sim 1$. In fact, $\left|\chi_{\mathbf{k}_{i}}(R=0)\right|^{2} /\left|\chi_{\mathbf{k}_{i}}(R \sim 1)\right|^{2}>10^{6}$ for low collision energies, and hence, we conclude that proton-antiproton annihilation dominates over electron-positron annihilation. A more detailed evaluation of the latter process is underway [27].

\section{THE ELASTIC CROSS SECTION AND LOW-ENERGY BEHAVIOR} tion

The elastic cross section can be extracted using the rela-

$$
\sigma^{\mathrm{el}}\left(k_{i}\right)=\frac{\pi}{k_{i}^{2}}\left|1-S_{i i}\left(k_{i}\right)\right|^{2},
$$

where $S_{i i}$ is the scattering matrix element related to the phase shift via

$$
S_{i i}\left(k_{i}\right)=\exp \left[2 i \delta_{0}\left(k_{i}\right)\right]
$$

Here, as above, we only take $s$-wave scattering into account. The real part of the phase shift has been determined by fitting the numerical radial solutions $f_{0}\left(k_{i}, R\right)$ at large $R$ to the form $N \sin \left(k_{i} R+\operatorname{Re}\left\{\delta_{0}\left(k_{i}\right)\right\}\right)$. The elastic cross section obtained in this way does not, however, take into account the presence of inelastic scattering.

In the presence of inelastic scattering, the phase shift $\delta_{0}$ is a complex quantity. The absorption factor $\left|S_{i i}\right|$ is related to the imaginary part of the phase shift through [21]

$$
\left|S_{i i}\right|=\exp \left(-2 \operatorname{Im} \delta_{0}\right) .
$$

Unitarity gives the restriction $0 \leqslant\left|S_{i i}\right| \leqslant 1$. We may extract the imaginary part of the phase shift from the sum of the rearrangement and in-flight annihilation cross sections

$$
\sigma^{\text {inel }}=\frac{\pi}{k_{i}^{2}}\left(1-\left|S_{i i}\right|^{2}\right)=\frac{\pi}{k_{i}^{2}}\left[1-\exp \left(-4 \operatorname{Im} \delta_{0}\right)\right] .
$$

We may then correct the elastic cross section using the results for the inelastic-scattering cross section. The $s$-wave elastic cross section with and without the correction for inelasticity is presented in Fig. 6. The effect of this correction is small. The exceptions are the dips in the cross section at

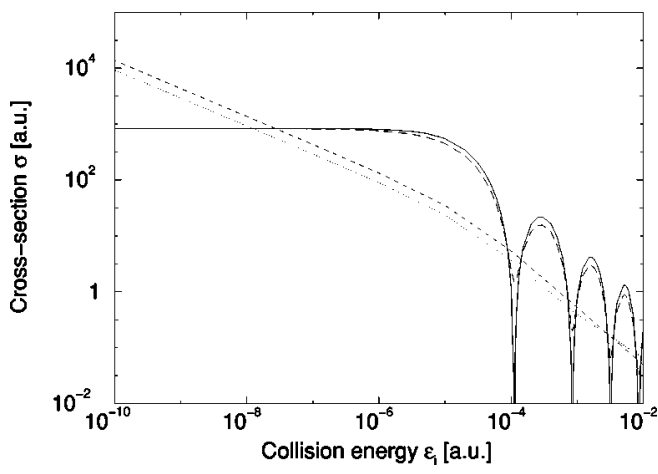

FIG. 6. Cross sections for the $\mathrm{H}-\overline{\mathrm{H}}$ system: elastic cross section obtained from the real part of the phase shift only (solid), elastic cross section including correction for the presence of inelastic scattering (long dashed), rearrangement cross section (dotted), and proton-antiproton annihilation in flight (dashed). At low energies, the elastic cross section is 823 without the correction for inelastic scattering, and 829 including this correction, while the low-energy behavior of $\sigma^{\text {rearr }}$ is $0.09 / \sqrt{\epsilon_{i}}$, and $\sigma_{a}^{p \bar{p}} \sim 0.14 / \sqrt{\epsilon_{i}}$.

energies where the real part of the phase shift goes through zero, which are smoothed out by the presence of inelastic scattering.

As seen in Fig. 6, in the low-energy limit, the elastic cross section is constant. This is to be expected from the general theory of scattering of slow particles. At low energies, the phase shift may be expanded as [13]

$$
k_{i} \cot \delta_{0}\left(k_{i}\right)=-\frac{1}{a}+\frac{1}{2} r_{\mathrm{eff}} k_{i}^{2}+\ldots
$$

where $a$ is the scattering length, and $r_{\text {eff }}$ is the effective range. In the presence of inelastic scattering, the scattering length, just as the phase shift, is a complex quantity $a=\alpha$ $-i \beta$ [22]. The scattering matrix element $S_{i i}\left(k_{i}\right)$ can be expanded to first order in $k_{i}$, and related to the scattering length by

$$
S_{i i}\left(k_{i}\right) \simeq 1+2 i \delta_{0}\left(k_{i}\right) \simeq 1-2 i k_{i}(\alpha-i \beta) .
$$

The real part $\alpha$ of the scattering length and the effective range were obtained by fitting them to the previously obtained real part of the phase shifts according to Eq. (49) in the energy region $10^{-7} \leqslant \varepsilon_{i} \leqslant 10^{-10}$. This procedure gave $\alpha$ $=8.1$ and $r_{\text {eff }}=7.1$. The relation of the real part of the scattering length to the hadronic wave function is illustrated in Fig. 7.

The imaginary part $\beta$ of the scattering length has been obtained from its relation to the inelastic cross section. Substituting Eq. (50) into Eq. (48) gives the imaginary component of the scattering length in terms of the inelastic cross section

$$
\beta=\frac{k_{i}}{4 \pi} \sigma^{\text {inel }}
$$

For low energies, the sum of the inelastic cross sections behave as $0.23 \epsilon_{i}^{-1 / 2}$, and therefore shows the energy depen- 


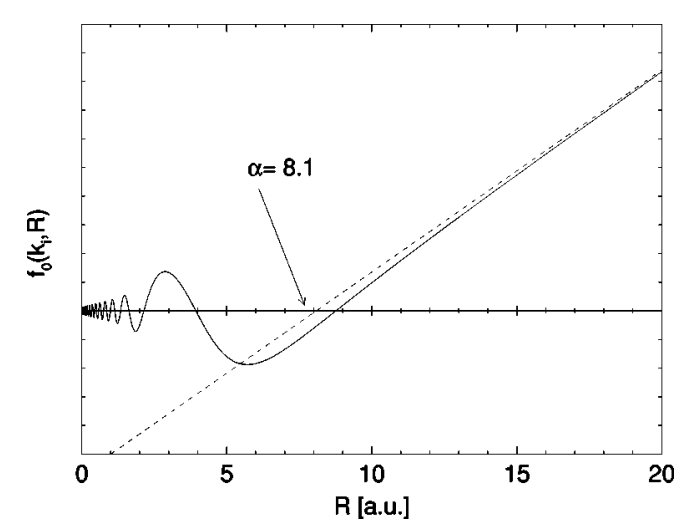

FIG. 7. Scattering length $\alpha=8.1$ for the $\mathrm{H}-\overline{\mathrm{H}}$ collisions and its relation to the hadronic scattering wave $f_{0}\left(k_{i}, R\right)\left(\epsilon_{i}=10^{-10}\right)$.

dence expected from Wigner's threshold law (see Fig. 3). Using this form in Eq. (51) we find $\beta=0.8$. The corrected low-energy limit of the elastic cross section is then

$$
\lim _{k_{i} \rightarrow 0} \sigma^{\mathrm{el}}\left(k_{i}\right)=4 \pi|a|^{2}=4 \pi\left(\alpha^{2}+\beta^{2}\right),
$$

which tends to the constant value $\sigma^{\mathrm{el}}=829$. Comparing this value to the inelastic cross sections, we find that elastic scattering dominates for energies larger than $7.9 \times 10^{-8}$. This means, for instance, that sympathetic cooling of antihydrogen via collisions with cold hydrogen will not be possible once the antihydrogen atoms are slowed down to this threshold energy, corresponding to a kinetic energy of $0.05 \mathrm{~K}$ (assuming the hydrogen atoms to be at rest).

Our results differ significantly from the results of a recent calculation by Sinha and Ghosh [23], which yielded an elastic-scattering length of about 4.8 (estimated from their graph) and an elastic cross section of $\sigma^{\mathrm{el}} \simeq 280$ in the limit of zero collision energy. The results were obtained using a close-coupling approach, with a basis that included a number of states of the hydrogen atom, but only the ground-state configuration of the antihydrogen atom. In view of the importance of the electron-positron correlation, we believe that this basis is too restrictive for an adequate description of low-energy scattering. In particular, it does not lead to the attractive van der Waals interaction.

For bound $\mathrm{H}-\overline{\mathrm{H}}$, one can introduce $\kappa=-i k_{i}$, which is related to the binding energy $\mathcal{E}_{b}$ of a state below threshold through [13]

$$
\kappa=\sqrt{2 \mu_{i}\left|\mathcal{E}_{b}\right|} .
$$

The bound states appear at the poles of the $S$ matrix. In terms of the phase shift this condition translates to

$$
i \kappa \cot \delta_{0}(i \kappa)=-\kappa .
$$

Energies of bound states close to threshold may be obtained putting the expansion Eq. (49) into Eq. (54). For H- $\overline{\mathrm{H}}$ scattering we did, however, find that due to the large effective range, $r_{\mathrm{eff}} \simeq \alpha$, the resulting equation has no real solutions. This was confirmed by direct integration of the numerical potential, giving a most loosely bound state with binding energy $\mathcal{E}_{b}=4.6 \times 10^{-5}$. This energy corresponds to $\kappa=0.29$, which is outside the range of validity of the expansion Eq. (49). An attempt to extend Eq. (49) to higher energies by including a $k^{4}$ term in the expansion, resulted in the binding energy $\mathcal{E}_{b}=5.6 \times 10^{-5}$, in approximate agreement with the result from numerical integration.

The relatively large binding energy of the state nearest to threshold can be understood from the form of the potential $V_{i}(R)$ plotted in Fig. 2. Here we compare to the potential of pure Coulombic attraction between the hadrons, which at short distances is a good approximation to $V_{i}(R)$. The Coulomb potential supports an infinite series of near-threshold states. The $\mathrm{H}-\overline{\mathrm{H}}$ potential does, however, bend off quite sharply at $R \sim 1$, and tends to a different threshold -1 , which greatly reduces the number of near-threshold states supported.

In the presence of a loosely bound state created by the scattering potential, the low-energy elastic cross section is given by [13]

$$
\sigma^{\mathrm{el}}=\frac{2 \pi}{\mu_{i}} \frac{1+r_{\mathrm{eff}} \kappa}{\epsilon_{i}+\left|\mathcal{E}_{b}\right|} .
$$

In the present case, this formula is not strictly valid because the most loosely bound state falls outside the range of validity of the expansion Eq. (49). Nevertheless, as is seen from Fig. 6, the elastic cross section still changes character from the typical low-energy behavior at $\epsilon_{i} \simeq 10^{-5} \sim\left|\mathcal{E}_{b}\right|$ as predicted by Eq. (55). A similar behavior was found for the annihilation in flight, as was discussed in Sec. III A.

\section{COLLISIONAL COOLING OF ANTIHYDROGEN}

The loss of antihydrogen atoms due to collisions with hydrogen atoms is described by

$$
\frac{d n^{\overline{\mathrm{H}}}}{d t}=-\sigma^{\mathrm{inel}} v n^{\mathrm{H}} n^{\overline{\mathrm{H}}},
$$

where $n^{\mathrm{H}}$ is the density of hydrogen, $n^{\overline{\mathrm{H}}}$ the density of antihydrogen, and $v$ the relative velocity of the atoms. As was shown in Sec. IV the rate of inelastic collisions, leading to loss of antihydrogen, dominates over the rate of elastic collisions for collisional energies less than $7.9 \times 10^{-8}$. We shall examine the solutions to Eq. (56) in three limiting cases; (i) $n^{\mathrm{H}} \gg n^{\overline{\mathrm{H}}}$, (ii) $n^{\mathrm{H}} \ll n^{\overline{\mathrm{H}}}$, and (iii) $n^{\mathrm{H}}=n^{\overline{\mathrm{H}}}$.

In case (i) the high density of hydrogen atoms is not significantly reduced by the collisions with antihydrogen, and is hence, approximately constant. If the hydrogen and antihydrogen atoms have different initial temperatures, the elastic scattering will lead to thermalization. In the present case, where $n^{\mathrm{H}} \gg n^{\overline{\mathrm{H}}}$, the final temperature of both types of atoms will equal the initial temperature of hydrogen. Cold hydrogen atoms may hence be used for the cooling of antihydrogen atoms, provided that the losses are not too severe. This process has been analyzed in Ref. [5], but with cross sections different from ours. 


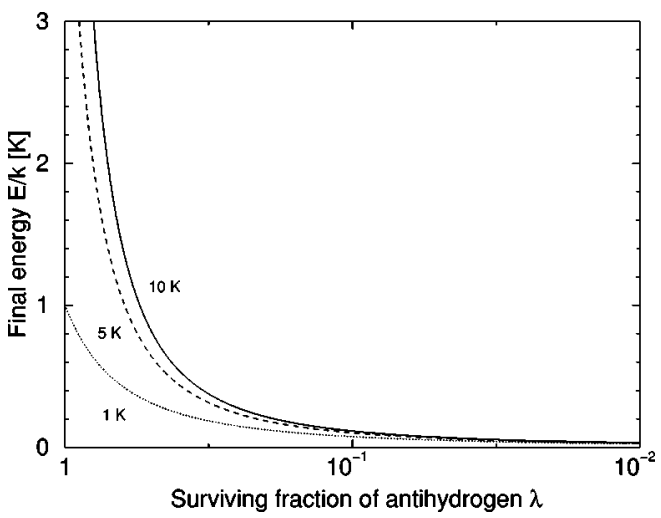

FIG. 8. Cooling of antihydrogen atoms due to elastic collisions with ultracold hydrogen as a function of the loss of antihydrogen atoms. Initial energy of the antihydrogen $E_{0}=10 \mathrm{~K}$ (solid), $E_{0}$ $=5 \mathrm{~K}$ (dashed), and $E_{0}=1 \mathrm{~K}$ (dotted).

Let $\lambda$ be the fraction of antihydrogen atoms that remain after a time $t\left[\lambda(t)=n^{\overline{\mathrm{H}}}(t) / n^{\overline{\mathrm{H}}}(0)\right]$. The relative velocity $v$ is approximately given by the velocity of the antihydrogen in the laboratory frame, since the hydrogen atoms have a much lower temperature. Hence, $v=\left(2 E / m_{p}^{-}\right)^{1 / 2}=\left(E / \mu_{i}\right)^{1 / 2}$, where $E$ is the kinetic energy of the antihydrogen atoms in the laboratory frame. Assuming also that $E \lesssim 10^{-5}$, so that the low-energy forms of the cross sections are valid, integration of Eq. (56) gives

$$
\lambda(t)=\exp \left(-\frac{4 \pi \beta n^{\mathrm{H}} t}{\mu_{i}}\right) .
$$

The antihydrogen atoms will loose half of their energy in each binary collision. The loss of kinetic energy of an antihydrogen atom through repeated elastic collisions with hydrogen atoms is then given by

$$
\frac{d E}{d t}=-n^{\mathrm{H}} v \sigma^{\mathrm{el}} \frac{E}{2}=-2 \pi n^{\mathrm{H}} \alpha^{2} E^{3 / 2} / \mu_{i}^{1 / 2} .
$$

If $E_{0}$ and $v_{0}$ are the initial kinetic energy and velocity of the antihydrogen atoms, the solution of Eq. (58) is

$$
E(t)=E_{0}\left\{1+\pi n^{\mathrm{H}} \alpha^{2} v_{0} t\right\}^{-2} .
$$

Inverting the relation (57), the time variable in Eq. (59) can be substituted by the fractional loss of antihydrogen

$$
E(\lambda)=E_{0}\left\{1-\frac{\alpha^{2} \sqrt{E_{0} \mu_{i}}}{4 \beta} \ln \lambda\right\}^{-2} .
$$

The result is independent of the density of hydrogen atoms. Using the values of $\alpha$ and $\beta$ obtained in Sec. IV, we have in Fig. 8 plotted the energy after cooling as a function of the loss of antihydrogen for initial energy $E_{0}=1,5$, and $10 \mathrm{~K}$. We see that largely independently of the initial energy, the antihydrogen is cooled down to $0.05-0.1 \mathrm{~K}$, while loosing $90 \%$ of the atoms. The result agrees well with the estimate in Sec. IV based on the crossing of the elastic and inelastic cross sections. Below this limit, further cooling only occurs at the expense of a very high loss of antiatoms.
It is also interesting to look at the time scales for cooling. The cooling rate depends on the density of hydrogen atoms. Hydrogen atoms have been cooled down to $100 \mu \mathrm{K}$ with densities up to $6 \times 10^{13} \mathrm{~cm}^{-3}$ [24]. According to Eq. (59), for this density of hydrogen atoms, the cooling of antihydrogen from 10 to $0.1 \mathrm{~K}$ would take about $0.6 \mathrm{~ms}$, independently of the initial density of antihydrogen atoms as long as it is much less than that of hydrogen atoms.

Case (ii), $n^{\mathrm{H}} \ll n^{\overline{\mathrm{H}}}$, corresponds to the situation where some antihydrogen in a trap is lost due to a small background density of hydrogen. The storage times for antihydrogen will depend on the temperature and the densities of atoms and antiatoms. Assuming a constant background density due to hydrogen atoms leaking into the trap, the decrease in the number of antiatoms is again given by Eq. (57). Storage times for antiprotons at $4.2 \mathrm{~K}$ have been measured to be on the order of months or longer [25]. A recent calculation of the rearrangement cross section in hydrogen-antiproton scattering [18] gave a result about 40 times larger than our result for hydrogen-antihydrogen scattering. Hence, under vacuum conditions similar to those in Ref. [25] $\left(<250 \mathrm{atoms} / \mathrm{cm}^{3}\right.$ or pressure $<10^{-16}$ torr) storage times of antihydrogen should be on the order of years.

If hydrogen atoms cannot leak into the trap, any hydrogen atoms present when the trap is loaded with antihydrogen will of course be removed through annihilation. Hence, the stored antihydrogen is "self-cleaning," and storage times are, as far as annihilation is concerned, indefinite.

In case (iii), we consider the lifetime of a cloud consisting of hydrogen and antihydrogen in equal proportions. For example, spectroscopy on the $1 s-2 s$ line could perhaps be done on a sample containing a mixture of hydrogen and antihydrogen atoms, provided that the atoms and antiatoms can coexist for a sufficiently long time. It would then not be necessary to determine the absolute energy of the line. Any difference between matter and antimatter, implying a violation of the $C P T$ symmetry, would manifest itself as a double peak in the $1 s-2 s$ line. It should be much easier to distinguish two nearly overlapping peaks in a single data set, rather than to compare the absolute energies obtained from two separate experiments on hydrogen and on antihydrogen, respectively.

Assuming that $n^{\overline{\mathrm{H}}}=n^{\mathrm{H}}=n$ in Eq. (56), we obtain for low energies the solution

$$
n(t)=\frac{n_{0}}{1+4 \pi \beta n_{0} t / \mu_{i}},
$$

where $n_{0}$ is the initial density. Hence, the time it takes before the density is halved is

$$
t_{1 / 2}=\frac{\mu_{i}}{4 \pi \beta n_{0}} .
$$

For an antihydrogen hydrogen density of $10^{7}$ atoms $/ \mathrm{cm}^{3}$ we obtain $t_{1 / 2}=26 \mathrm{~min}$. Hence, it is possible for antihydrogen and hydrogen to coexist in a trap for a considerable amount of time. 


\section{CONCLUSIONS AND DISCUSSION}

The elastic cross section for the hydrogen-antihydrogen collisions turns out to be rather large. The scattering length is 8.1, which is 18 times larger than the hydrogen-hydrogen scattering length (the latter is 0.45 for collisions in the singlet state [26]). Since the effective atom-atom interaction at large separations is the same for both systems the large difference in scattering length must be due to the very different interaction potential (attractive vs repulsive) at short distances. As expected, the elastic cross section tends for low collision energies to a constant value (see Fig. 7), determined by the scattering length. The limiting value of the elastic cross section is $\sigma^{\mathrm{el}}=829$. The low-energy scattering formulas remain valid up to an energy equal to the binding energy of the most loosely bound state of the scattering potential. The binding energy of this state is $\mathcal{E}_{b}=4.6 \times 10^{-5}$.

We find that loss due to in-flight annihilation (36) and due to the rearrangement reaction (1) are comparable in magnitude. The sum of the inelastic cross sections become larger than the elastic cross section for energies below $7.9 \times 10^{-8}$. This means that the cooling of antihydrogen via collisions with cold hydrogen is inefficient, once the antihydrogen atoms are slowed down below $0.05 \mathrm{~K}$. Our estimates based on the rate equations suggest that further cooling is possible only at the expense of large density losses. This is due to the small value of the ratio of the cross sections for elastic and inelastic scattering. The low-energy reaction rate is for annihilation in flight $\lambda^{\text {ainfl }}=1.2 \times 10^{-17} \mathrm{~m}^{3} \mathrm{~s}^{-1} n^{\mathrm{H}}$ and for rear- rangement is $\lambda^{\text {rearr }}=7.6 \times 10^{-18} \mathrm{~m}^{3} \mathrm{~s}^{-1} n^{\mathrm{H}}$. At the low densities that are feasible in experiments, this rate is low, leading to storage times for mixtures of hydrogen and antihydrogen of the order of minutes.

While trying to find the relevant parameters for the coexistence of hydrogen and antihydrogen and for the prospects of collisional cooling of antihydrogen, we found that the $\mathrm{H}-\overline{\mathrm{H}}$ system is a very instructive laboratory for the studies of cold collisions, with many interesting features due to the attractive nature of the short-range potential, the competition of different scattering processes, and the influence of the most loosely bound state below the $\mathrm{H}-\overline{\mathrm{H}}$ dissociation threshold. In particular, the process of radiative association into a bound $\mathrm{H}-\overline{\mathrm{H}}$ state, and its possible use as a diagnostic, is the subject of another paper [28].

\section{ACKNOWLEDGMENTS}

The work of A.D. was supported by the Chemical Sciences, Geosciences, and Biosciences Division of the Office of Basic Energy Sciences, Office of Science, U.S. Department of Energy. P.F. acknowledges (with gratitude) the support from the Swedish National Research Council (NFR) and from the National Science Foundation through a grant for the Institute of Theoretical Atomic and Molecular Physics (ITAMP) at Harvard University and Smithsonian Astrophysical Observatory.
[1] D. L. Morgan and V. W. Hughes, Phys. Rev. D 2, 1389 (1970).

[2] B. R. Junker and J. N. Bardsley, Phys. Rev. Lett. 28, 1227 (1972).

[3] D. L. Morgan and V. W. Hughes, Phys. Rev. A 7, 1811 (1973).

[4] W. Kołos, D. L. Morgan, Jr., D. M. Schrader, and L. Wolniewicz, Phys. Rev. A 11, 1792 (1975).

[5] G. V. Shlyapnikov, J. T. M. Walraven, and E. L. Surkov, Hyperfine Interact. 76, 31 (1993).

[6] M. H. Holzscheiter and M. Charlton, Rep. Prog. Phys. 62, 1 (1999).

[7] E. A. G. Armour, J. M. Carr, and V. Zeman, J. Phys. B 31, L679 (1998).

[8] M. H. Holzscheiter et al., CERN Technical Report No. SPSLC 96-47/P302, CERN (unpublished).

[9] M. H. Holzscheiter and M. Charlton, Rep. Prog. Phys. 62, 1 (1999).

[10] G. Gabrielse et al., CERN Technical Report No. SPSC 97-8/ P306 (unpublished).

[11] T. Azuma et al., CERN Technical Report No. SPSC 97-19/ P307 (unpublished).

[12] P. Froelich, S. Jonsell, A. Saenz, B. Zygelman, and A. Dalgarno, Phys. Rev. Lett. 84, 4577 (2000). In this work, a fourtimes larger value for the rearrangement cross section was given. The discrepancy was due to a numerical error that has now been corrected.
[13] L. D. Landau and E. Lifshitz, Quantum Mechanics (Pergamon, Oxford, 1965).

[14] E. A. G. Armour and T. Zeman, Int. J. Quantum Chem. 74, 645 (1999).

[15] A. S. Wightman, Phys. Rev. 72, 521 (1950).

[16] Z.-C. Yan, J. F. Babb, A. Dalgarno, and G. W. F. Drake, Phys. Rev. A 54, 2824 (1996).

[17] P. Froelich, B. Jeziorski, W. Kołos, H. Monkhorst, A. Saenz, and K. Szalewicz, Phys. Rev. Lett. 71, 2871 (1993).

[18] A. Yu. Voronin and J. Carbonell, Phys. Rev. A 57, 4335 (1998).

[19] C. J. Batty, Nucl. Phys. A 601, 425 (1996).

[20] A. B. Migdal, A. M. Perelemov, and V. S. Popov, Yad. Fiz. 14, 874 (1971) [ Sov. J. Nucl. Phys. 14, 488 (1972)].

[21] C. Joachain, Collision Theory (North-Holland, Amsterdam, 1975).

[22] R. C. Forrey, N. Balakrishnan, V. Kharchenko, and A. Dalgarno, Phys. Rev. A 58, R2645 (1998).

[23] P. K. Sinha and A. S. Ghosh, Europhys. Lett. 49, 558 (2000).

[24] C. L. Cesar et al., Phys. Rev. Lett. 77, 255 (1996).

[25] G. Gabrielse et al., Phys. Rev. Lett. 65, 1317 (1990).

[26] M. Jamieson and A. Dalgarno, J. Phys. B 31, L219 (1998).

[27] S. Eriksson, A. Saenz, S. Jonsell, and P. Froelich (unpublished).

[28] B. Zygelman, A. Saenz, P. Froelich, S. Jonsell, and A. Dalgarno, Phys. Rev. A 63, 052722 (2001). 